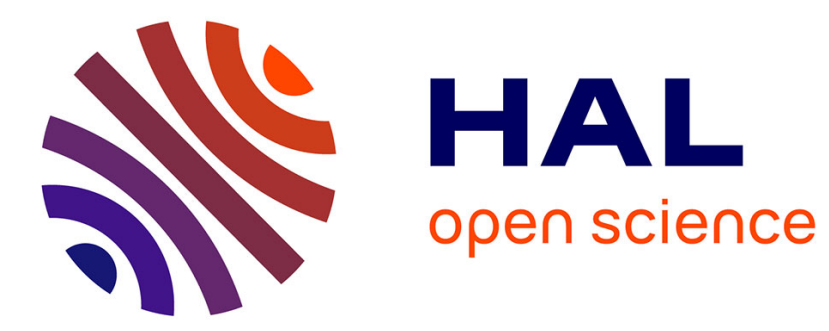

\title{
3D imaging for underfoliage targets using L-band multibaseline polinsar data and sparse estimation methods
}

Yue Huang, Jacques Levy Vehel, Laurent Ferro-Famil, Andreas Reigber

\section{- To cite this version:}

Yue Huang, Jacques Levy Vehel, Laurent Ferro-Famil, Andreas Reigber. 3D imaging for underfoliage targets using L-band multibaseline polinsar data and sparse estimation methods. IGARSS 2016 - IEEE International Geoscience and Remote Sensing Symposium, Jul 2016, Beijing, China. 10.1109/igarss.2016.7729373 . hal-01418435

\author{
HAL Id: hal-01418435 \\ https://hal.inria.fr/hal-01418435
}

Submitted on 19 Dec 2016

HAL is a multi-disciplinary open access archive for the deposit and dissemination of scientific research documents, whether they are published or not. The documents may come from teaching and research institutions in France or abroad, or from public or private research centers.
L'archive ouverte pluridisciplinaire $\mathbf{H A L}$, est destinée au dépôt et à la diffusion de documents scientifiques de niveau recherche, publiés ou non, émanant des établissements d'enseignement et de recherche français ou étrangers, des laboratoires publics ou privés. 


\title{
3D IMAGING FOR UNDERFOLIAGE TARGETS USING L-BAND MULTIBASELINE POLINSAR DATA AND SPARSE ESTIMATION METHODS
}

\author{
Yue Huang ${ }^{1}$, Jacques Levy-Vehel ${ }^{1}$, Laurent Ferro-Famil ${ }^{2}$, Andreas Reigber ${ }^{3}$ \\ ${ }^{1}$ INRIA Bretagne-Atlantique, France \\ ${ }^{2}$ University of Rennes 1, France \\ ${ }^{3}$ Microwaves and Radar Institute, German Aerospace Center (DLR), Germany.
}

\begin{abstract}
SAR imaging of concealed targets beneath the canopies has to face a complex mixture of diverse scattering mechanisms. To characterize this complex scattering environment, nonparametric tomographic estimators are more robust to focusing artefacts but limited in resolution. Parametric tomographic estimators provide better vertical resolution but fail to adequately characterize continuously distributed volumetric scatterers such as forest canopies. To overcome these limitations, this paper addresses a new wavelet-based sparse estimation method for 3D imaging and characterization for underfoliage objects. The effectiveness of this new approach is demonstrated by using L-band Multi-Baseline PolInSAR Data over Dornstetten, Germany.
\end{abstract}

Index Terms - SAR tomography, underfoliage, sparse estimation

\section{INTRODUCTION}

Hybrid environments refer to a scenario of objects embedded in a host natural environment (e.g. forests). Their scattering patterns consist of a complex mixture of diverse scattering mechanisms, like the volume scattering from the canopy, double bounce reflection between the ground and under-foliage objects as well as between an object and trunks, surface scattering from the underlying ground, etc. The resulting SAR information is characterized by a strong complexity, which makes SAR image analysis difficult by means of PolInSAR data (due to the single-baseline configuration). Multi-baseline PolInSAR techniques can be applied to reconstruct the associated scattering responses and polarimetric patterns. In [1], using single polarization tomograms, the forest profile and truck shape were both extracted using Capon's spectral estimation approach. However, due to the limited spectral resolution and sidelobe suppression of such an approach, parametric and fully polarimetric tomographic approaches are expected to show significantly improved features of under-foliage objects and forests as it has been shown in [2]. In [1, 2], the applied spectral estimation methods are either nonparametric or parametric. It is known that nonparametric approaches are in general more robust to focusing artefacts, whereas parametric approaches are characterized by a better vertical resolution. It has been shown $[3,4]$ that the performance of these spectral analysis approaches is conditioned by the nature of the scattering response of the observed objects.

The scattering power from forest canopy in elevation can be characterized by continuous spectrum, since volumic scatterers are continuously distributed in vertical. Scattering responses from the objects or ground are considered to be localized discretely in the vertical direction. The underfoliage object with a deterministic scattering response embedded in the surrounding distributed environments, should be characterized by a mixed spectrum. Considering Conventional tomographic estimators, either nonparametric methods(e.g.Capon, Beamforming) or parametric methods (e.g. Maximum likelihood)are limited to one type of spectrum or the other, due to the lack of adaptation to the mixed spectrum. Semiparametric methods like sparse estimation methods, have been used for compressive sensing over urban areas in [5] and over forested areas in [6]. However for this scenario of complex mixed scattering environment, a new wavelet-based approach is proposed, adapted to characterize the hybrid nature of the observed environment. The effectiveness of the proposed method is demonstrated by using the L-band multibaseline InSAR data acquired over the test site of Dornstetten, Germany.

\section{TOMOGRAPHIC SIGNAL MODEL}

The MB-InSAR configuration consists of $M$ acquisition positions, each pair of which are separated by a baseline. Considering an azimuth-range resolution cell that contains $K$ backscattering contributions from scatterers located at different heights $\mathbf{z}=\left[z_{1}, \ldots, z_{K}\right]$, the received data vector, $\mathbf{y} \in \mathbb{C}^{M \times 1}$, can be formulated as follows:

$$
\mathbf{y}(l)=\sum_{i=1}^{K} \mathbf{a}\left(z_{i}\right) s_{i}(l)+\mathbf{n}(l)
$$

where $l=1, \ldots, L$ indicates one of the $L$ independent realizations of the signal acquisition. The complex additive noise 
$\mathbf{n} \in \mathbb{C}^{M}$ is assumed to be Gaussianly distributed with zero mean and variance $\sigma_{n}^{2}$ and to be white in time and space. The steering vector $\mathbf{a}(z)$ contains the interferometric phase information associated to a source located at the elevation position $z$ above the reference focusing plane and is given by:

$$
\mathbf{a}(z)=\left[1, \exp \left(j k_{z_{2}} z\right), \ldots, \exp \left(j k_{z_{M}} z\right)\right]^{T}
$$

where $k_{z_{j}}=\frac{4 \pi}{\lambda} \frac{B_{\perp_{j}}}{r_{1} \sin \theta}$ is the two-way vertical wavenumber between the master and the $j$ th acquisition tracks. The carrier wavelength is represented by $\lambda$, whereas $\theta$ stands for the incidence angle and $r_{1}$ is the slant range distance between the master track and the scatterer. As $k_{z_{j}}$ is calculated from acquisition system parameters, $\mathbf{a}($.$) is a known function but the$ source elevation $z$ is unknown. The source signal vector, $\mathbf{s}=$ $\left[s_{1} \ldots s_{K}\right] \in \mathbb{C}^{K}$, contains the unknown complex backscattering coefficients of the $K$ source scatterers. We are interested in recovering the power distribution in elevation (i.e. tomographic profile), $\mathbf{x}=\left[\left|s_{1}\right|^{2}, \ldots,\left|s_{K}\right|^{2}\right] \in \mathbb{R}^{K}$, from the observed data $[\mathbf{y}(1), \ldots \mathbf{y}(L)] \in \mathbb{C}^{M \times L}$. Conventionally, $K<M$ scatterers are assumed to be located within one resolution cell. However, the forest canopy consists of a large number of elementary scatterers continuously distributed in the vertical direction. To characterize the object embedded in a forest environment, it is reasonable to assume $K>>M$. In order to solve this underdetermined problem, a wavelet-based sparse estimation method is proposed.

\section{TOMOGRAPHIC ESTIMATION}

In the frame of urban remote sensing, the $l_{1}$ norm regularization has been used for tomographic SAR inversion by [5]. This compressive approach can efficiently localize the pointlike scatterers in the vertical direction, but can not directly deal with volumic media because the volumic scatterers are not distributed sparsely in the vertical domain. In [6], a wavelet-based compressed sensing technique has been developed for SAR tomography of forested areas and it provided undeniable performance for forest characterization. However this approach lacks high resolution to characterize the objects beneath the canopy. For this purpose, a criterion is proposed and compactly written in a standard format of $l_{1}$ norm minimization with quadratic constraints

$$
\min _{\mathbf{x}}\|\mathbf{B} \mathbf{x}\|_{1} \text { subject to }\left\|\mathbf{A} \mathbf{\Lambda}_{\mathbf{x}} \mathbf{A}^{\dagger}-\widehat{\mathbf{R}}\right\|_{F} \leq \epsilon
$$

where

$$
\text { - } \mathbf{x}=\left[\begin{array}{l}
\mathbf{x}_{1} \\
\mathbf{x}_{2}
\end{array}\right] \in \mathbb{R}^{K}: \text { scattering power distribution in }
$$
elevation. $\mathbf{x}_{1} \in \mathbb{R}^{p}$ and $\mathbf{x}_{2} \in \mathbb{R}^{q}$ represent respectively scattering power distribution for the objects and canopy in elevation $(N=p+q)$.

$\underset{\text { - }}{\mathbf{B} \mathbf{x}}=\left[\begin{array}{cc}\mathbf{I}_{(p \times p)} & \mathbf{0} \\ \mathbf{0} & \mathbf{\Psi}\end{array}\right] \mathbf{x}=\left[\begin{array}{c}\mathbf{x}_{1} \\ \mathbf{\Psi}_{\mathbf{x}_{2}}\end{array}\right] \in \mathbb{R}^{K}$ : sparse
- $\mathbf{x}_{1} \in \mathbb{R}^{p}$ is sparse as the scattering responses of an object may be described with a few scatterers discretely localized in elevation.

- $\boldsymbol{\Psi}_{\mathbf{x}_{2}} \in \mathbb{R}^{q}$ represents the projection of canopy power distribution $\mathbf{x}_{2}$ onto an orthonormal wavelet basis $\Psi \in \mathbb{R}^{q \times q}$. The power distribution of canopy in elevation, $\mathbf{x}_{2}$, depicts continuous regular signal behaviors, so its projection onto wavelet basis, $\Psi_{\mathbf{x}_{2}}$, is sparse.

- $\boldsymbol{\Lambda}_{\mathbf{x}}=\operatorname{diag}(\mathbf{x}) \in \mathbb{R}^{N \times N}$.

- $\widehat{\mathbf{R}}=\frac{1}{L} \sum_{1}^{L} \mathbf{y}(l) \mathbf{y}^{\dagger}(l) \in \mathbb{R}^{M \times M}:$ sample covariance matrix of observed data.

- $\epsilon$ : tolerance parameter to control model mismatch and signal sparsity.

By minimizing the criterion (3), the solution $\widehat{x}$ is obtained that reconstructs the vertical power distribution of underfoliage objects. In practice, the problem in (3) is under SOCP formulation and can be solved by any convex optimization solver proposed in the field of sparse recovery estimation. In this paper, the CVX solver is implemented to solve this standard $l_{1}$ norm minimization, due to its implementation facility and compactness. The parameter $\epsilon$ is selected based on noise variance $\sigma_{n}^{2}$, so according the (3), $\epsilon$ should be $\propto \sqrt{M} \sigma_{n}^{2}$, e.g. for our applied data sets, we choose $\epsilon=4 \sqrt{M} \sigma_{n}^{2}$.

For the sparse estimation controlled by the user-selected parameter, the drawback is the source signal power leaks into the neighboring sampling intervals. This fact may be due to the inappropriate choice for the parameter $\epsilon$. If $\epsilon$ is chosen too small, then the measured data will be overfitted and the mainlobe of the source signal power spectrum may be splitted in several peaks, especially in the low SNR case. The other reason may be due to the off-grids effects and basis mismatch[7],[8]. This type of leakage happens more often to the estimated signal associated to the $\mathbf{I}$ basis [8], i.e. $\mathbf{x}_{1}$ in our criterion (3) . To solve this issue, a merging processing based on linear interpolation is used here, inspired from [7]. Candidate peaks with amplitude $>\max \left(\left|\widehat{\mathbf{x}}_{1}\right|\right) / 10$ can be selected from estimated reflectivity $\widehat{\mathbf{x}}_{1}$ for underfoliage objects or ground reflection. Taking a window with width $\Delta$ and centering at each peak location $z_{i}$, the neighboring peaks $\left[z_{i_{1}}, \ldots, z_{i_{d}}\right]$ located within $\left[z_{i}-\Delta / 2, z_{i}+\Delta / 2\right]$ are merged in such a way: $z_{m}=z_{i c}+\sum_{k=1}^{d} \mathbf{x}_{1}\left(z_{i}\right)\left(z_{i_{k}}-\right.$ $\left.z_{i c}\right) / \sum_{k=1}^{d} \mathbf{x}_{1}\left(z_{i}\right)$ with $z_{i c}=\left(z_{i_{1}}+\cdots+z_{i_{d}}\right) / d$. This method effectively merges splitted peaks within a filtering window $\Delta$ into one peak.

\section{EXPERIMENTAL RESULTS}

The tomographic analysis of a volumetric forested area and sub-canopy objects is led using polarimetic L-band airborne 

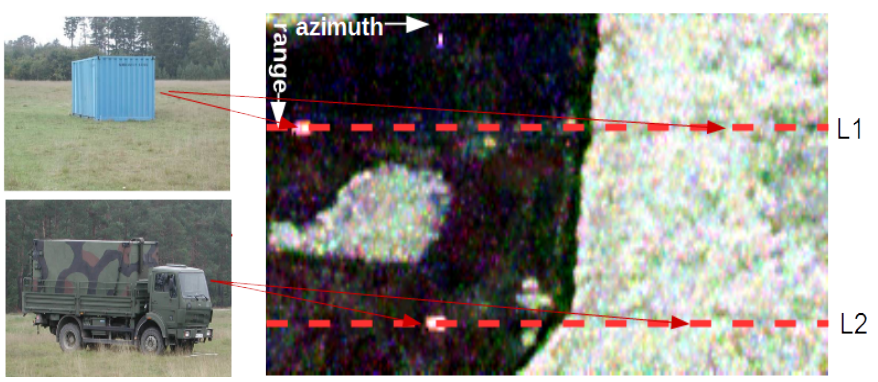

Fig. 1: Pauli image for test area where concealed objects are set

data acquired in September 2006 by the DLR's E-SAR system over the Dornstetten test site, Germany. Some objects like vehicles and containers have been set inside and outside the forests for the purpose of underfoliage imaging. 21 tracks are effectively used for the tomographic analysis. The horizontal baselines form a quasi-uniform linear array with an average baseline close to $20 \mathrm{~m}$ and the geometric vertical resolution is about $2 \mathrm{~m}$. This quasi-regular distribution provides $3 \mathrm{D}$ features with reduced sidelobe levels and hence facilitates the tomographic analysis. The test zone for underfoliage objects like trucks and containers is shown in Fig.1, where the containers and trucks are concealed in the forested areas.

Applying now both the conventional tomographic techniques and the proposed sparse estimation method to the test line (L1), the reflectivity tomograms are obtained in Fig.2. Two containers are quite visible in the Capon tomogram with strong backscattering power(shown in purple circles Fig.2(a)). However the height of containers is not able to accurately easily due to the sidelobes and poor vertical resolution. Using $n_{s}$ estimated by diagonal loading MOS technique in [9], the MUSIC tomogram Fig.2(b) depicts strong sidelobes for the container outside of forests. As a discrete spectral estimation method, the MUSIC tomogram only describes a part of scatterers of forest canopy. Using the proposed sparse estimation method with merging process, the tomogram in Fig.2(c) enjoys the spectral continuity for the distributed natural environment and the high resolution for the man-made objects. The concealed container can be seen by energetic reflectivities and its estimated height is around $2 \mathrm{~m}$. Also using the proposed method, the ground and underfoliage scattering contributions can be separated from complex distributed environments and their tomograms are respectively shown in Fig.2(d)(e).

The entire test line (L2) is estimated by diverse tomographic estimators and the resulting reflectivity tomograms are given in Fig.3. The Capon tomogram depicts trucks with a poor vertical resolution due to the nature of continuous spectrum (Fig.3(a)), although it can well charcterize the forest canopy. The MUSIC tomogram characterizes the uncovered truck with strong sidelobes in the vertical direction

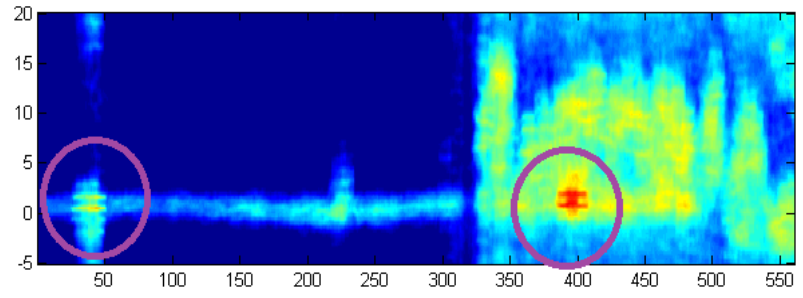

(a) Capon

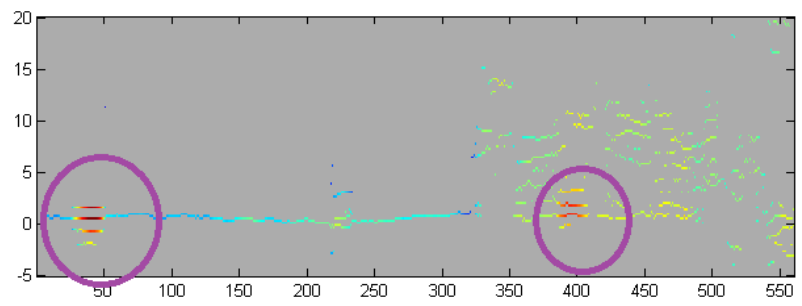

(b) MUSIC

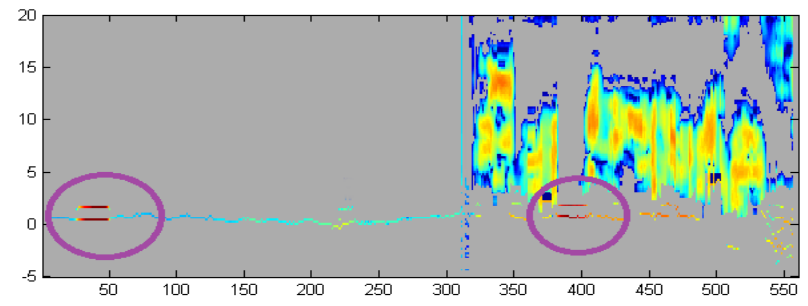

(c) Proposed method with merging

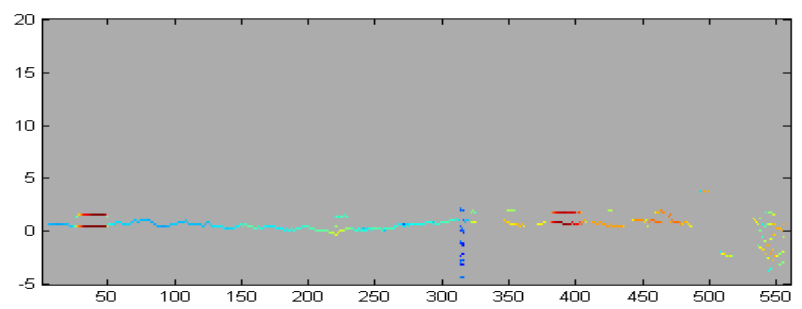

(d) Ground and underfoliage scattering $\left(\mathbf{x}_{1}\right)$ estimated by proposed method with merging

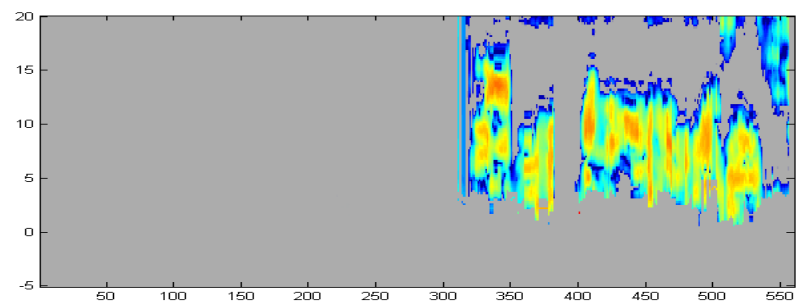

(e) Canopy power $\left(\mathbf{x}_{2}\right)$ estimated by proposed method with merging

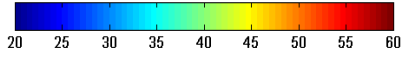

(f) $\mathrm{dB}$

Fig. 2: Reflectivity tomograms estimated by the proposed method with merging processing using $\mathrm{HH}$ data set over test line L1. (Gray background:zero reflectivity). 
and the forests with a limited number of scatterers (Fig.3(b)). Moreover, the underfoliage trucks are not visibly shown in the above two tomograms. Applying the proposed waveletbased method and merging process, the resulting tomogram demonstrates the continuous spectrum for the canopy and the high-resolution discrete spectrum for the ground and underfoliage object as shown in Fig.3(c). The scattering contributions from canopy and undercanopy are respectively shown in Fig.3(d)(e).

\section{REFERENCES}

[1] M.Nannini, R.Scheiber, and R.Horn, "Imaging of targets beneath foliage with sar tomography," Proc. EUSAR, pp. 1-4, June 2008.

[2] Y. Huang, L. Ferro-Famil, and C. Lardeux, "Polarimetric sar tomography of tropical forests at p and l-band.," Proc. IGARSS, pp. 1373-1376, July 2011.

[3] P. Stoica and A. Nehorai, "Performance study of conditional and unconditional direction-of-arrival estimation," IEEE Trans. Acoust. Speech Signal Processing, vol. ASSP-38, pp. 1783-1795, Oct 1990.

[4] Y.Huang and L. Ferro-Famil, "Building height estimation using multibaseline l-band sar data and polarimetric weighted subspace fitting methods," Polinsar proceeding, 2009.

[5] X. X. Zhu and R. Bamler, "Tomographic sar inversion by 11 -norm regularization-the compressive sensing approach," IEEE Transactions on Geoscience and Remote Sensing, vol. 48, no. 10, pp. 3839-3846, Oct 2010.

[6] E. Aguilera, M. Nannini, and A. Reigber, "Wavelet-based compressed sensing for sar tomography of forested areas," Geoscience and Remote Sensing, IEEE Transactions on, vol. 51, no. 12, pp. 5283-5295, Dec 2013.

[7] Z. Tan, P. Yang, and A. Nehorai, "Joint-sparse recovery in compressed sensing with dictionary mismatch," Computational Advances in Multi-Sensor Adaptive Processing (CAMSAP), 2013 IEEE 5th International Workshop on, pp. 248-251, Dec 2013.

[8] S.Xing, D. Dai, Y. Li, and X. Wang, "Polarimetric sar tomography using 12,1 mixed norm sparse reconstruction method," Progress In Electromagnetics Research, vol. 130, pp. 105-130, 2012.

[9] Y. Huang, L. Ferro-Famil, and A. Reigber, "Underfoliage object imaging using sar tomography and polarimetric spectral estimators," IEEE Transactions on Geoscience and Remote Sensing, vol. 50, no. 6, pp. 22132225, June 2012.

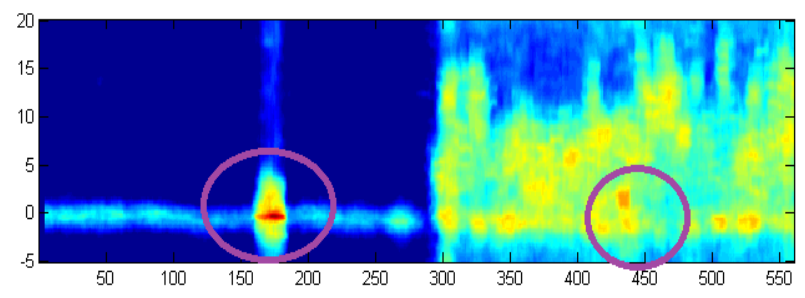

(a) Capon

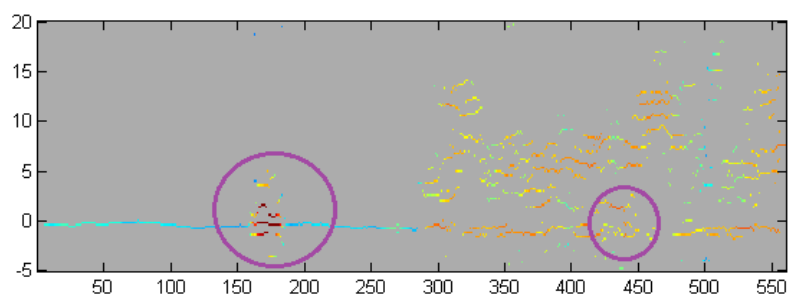

(b) MUSIC

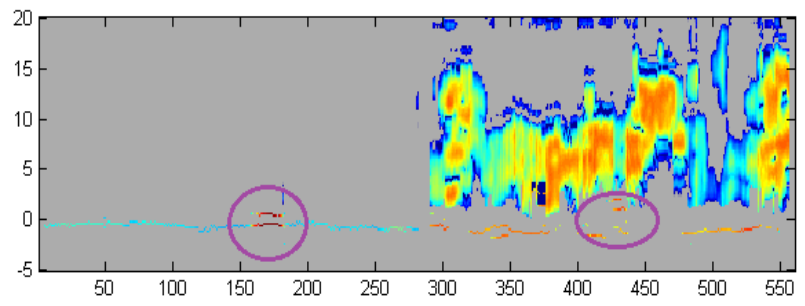

(c) Proposed method with merging

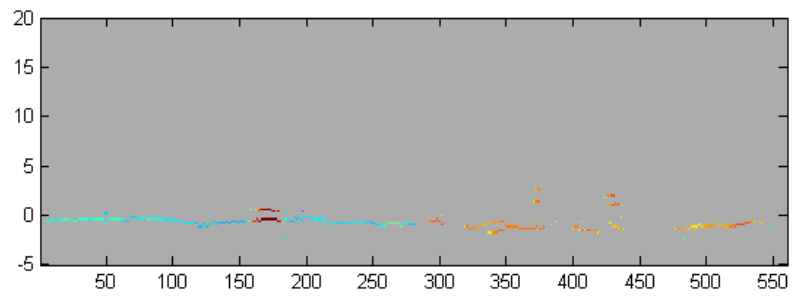

(d) Ground and underfoliage scattering $\left(\mathbf{x}_{1}\right)$ estimated by proposed method with merging

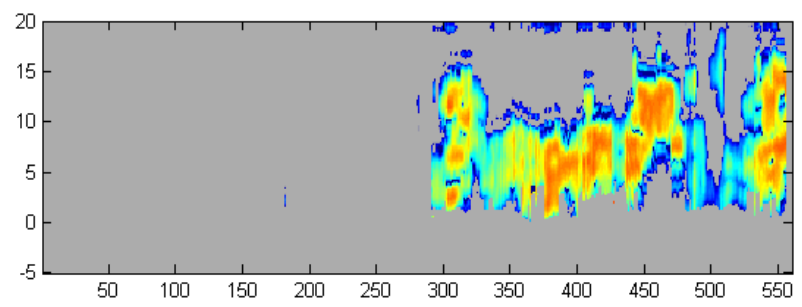

(e) Canopy power $\left(\mathbf{x}_{2}\right)$ estimated by proposed method with merging

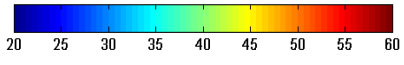

(f) $\mathrm{dB}$

Fig. 3: Reflectivity tomograms estimated by the proposed method with merging processing using $\mathrm{HH}$ data set over test line L2. (Gray background:zero reflectivity). 